\title{
Função sexual e saúde reprodutiva em mulheres adolescentes com lúpus eritematoso sistêmico juvenil
}

\author{
Clovis Artur Almeida da Silva ${ }^{1}$, Marília Vieira Febrônio², Eloísa Bonfá3, \\ Rosa Maria Rodrigues Pereira ${ }^{4}$, Elsa Aida Gay de Pereira ${ }^{5}$, Albertina Duarte Takiuiti ${ }^{6}$
}

\begin{abstract}
RESUMO
Objetivo: Avaliar a função sexual e a saúde reprodutiva em adolescentes com Lúpus Eritematoso Sistêmico Juvenil (LESJ) e compará-las com controles. Pacientes e Métodos: Cinquenta e duas pacientes com LESJ do sexo feminino foram avaliadas com dados demográficos, função sexual, exame ginecológico, ciclo menstrual, citologia cérvico-vaginal, características clínicas e tratamento. O grupo controle incluiu 52 mulheres pareadas por idade. Resultados: A média da idade foi similar nas pacientes com LESJ e controles $(16,7 \pm 1,94$ versus $16,13 \pm 2,16$ anos, $\mathrm{P}=0,92)$. A média da idade da menarca foi maior nas pacientes com LESJ (12,82 $\pm 1,62$ versus $11,55 \pm 1,45$ anos, $\mathrm{P}=0,0004)$. A frequência de atividade sexual foi significativamente menor nas pacientes com LESJ $(23 \%$ versus $60 \%, \mathrm{P}=0,0003)$. Em contraste, os percentuais de disfunção sexual, lubrificação vaginal reduzida, desempenho diminuído, orgasmo reduzido e insatisfação com a vida sexual foram significativamente maiores nas pacientes com LESJ ( $58 \%$ versus $23 \%, \mathrm{P}=0,03 ; 50 \%$ versus $16 \%, \mathrm{P}=0,046 ; 58 \%$ versus $23 \%, \mathrm{P}=0,03 \% ; 50 \%$ versus $16 \%, \mathrm{P}=0,046$; respectivamente). Por outro lado, nenhuma diferença foi observada nas pacientes com LESJ e controles em relação a dados demográficos, alterações pubertárias, anormalidades do ciclo menstrual e citologia cérvico-vaginal $(\mathrm{P}>0,05)$. Nenhuma diferença foi evidenciada nas pacientes com LESJ com e sem disfunção sexual em relação aos dados demográficos, alterações pubertárias, anormalidades do ciclo menstrual e citologia cérvico-vaginal, atividade da doença, dano cumulativo e tratamento $(\mathrm{P}>0,05)$. Conclusão: Este é o primeiro estudo que identificou disfunção sexual em adolescentes do sexo feminino com LESJ. Aspectos relacionados à sexualidade necessitam uma atenção especial dos profissionais de saúde que atendem adolescentes com lúpus.
\end{abstract}

Palavras-chave: saúde reprodutiva, função sexual, adolescente, hormônio, lúpus eritematoso sistêmico juvenil, sexo feminino.

\section{INTRODUÇÃO}

O Lúpus Eritematoso Sistêmico Juvenil (LESJ) é uma doença autoimune que apresenta maior prevalência no sexo feminino, habitualmente durante o período reprodutivo. ${ }^{1}$ Novas opções terapêuticas têm melhorado o prognóstico e também a qualidade de vida de adolescentes com esta doença, incluindo aspectos relacionados à saúde reprodutiva e à função sexual. ${ }^{1-3}$
A menarca é o marco inicial do começo do ciclo menstrual e do desenvolvimento pubertário. Estudos mostram que adolescentes com LESJ apresentam menarca atrasada quando comparadas com controles normais. ${ }^{4-6}$ Anormalidades menstruais após a primeira menstruação e ciclos de maior duração foram também mais frequentemente observadas nas pacientes com LESJ em relação aos controles, assim como amenorreia

\footnotetext{
Recebido em 23/04/2009. Aprovado, após revisão, em 10/10/2009. Os autores declaram ter recebido apoio do Conselho Nacional de Desenvolvimento Científico e Tecnológico - CNPQ (Grants 300248/2008-3 para CAAS e 305468/2006-5 para Eloísa Bonfá) e da Federico Foundation (para Eloísa Bonfá). Clovis Artur Almeida da Silva recebeu auxílio dos fundos remanescentes da SBR.

1. Professor Livre Docente do Departamento de Pediatria da FMUSP. Responsável pela Unidade de Reumatologia Pediátrica do Instituto da Criança (ICr) do Hospital das Clínicas (HC) da Faculdade de Medicina da Universidade de São Paulo (FMUSP)

2. Doutora em Reumatologia pela FMUSP. Médica Reumatologista Pediátrica

3. Professora Titular da Disciplina de Reumatologia do HC-FMUSP

4. Professor Livre Docente e Médica Assistente da Disciplina de Reumatologia do HC-FMUSP

5. Doutora em Ginecologia pela FMUSP. Médica Assistente do Departamento de Ginecologia da FMUSP

6. Doutora em Ginecologia pela FMUSP. Médica Responsável pelo Serviço de Ginecologia do Adolescente do Departamento de Ginecologia da FMUSP

Endereço para correspondência: Prof. Dr. Clovis Artur Almeida da Silva. Rua Araioses, 152/81. Vila Madalena - São Paulo - SP. CEP: 05442-010. Fax: (11)

3069-8503. E-mail: clovis.silva@icr.usp.br
} 
foi evidenciada em $11,7 \%$ das pacientes com LESJ em um recente estudo multicêntrico brasileiro. ${ }^{7}$

Outro aspecto relevante da saúde reprodutiva da adolescente com LESJ é a possibilidade de vaginites, displasias associadas ao papiloma vírus humano (HPV) e tumores cérvico-vaginais. Nós recentemente evidenciamos uma alta prevalência de candidíase vaginal e baixa frequência de displasias cérvico-vaginais associadas à infecção pelo papilomavírus humano (HPV) em adolescentes com LESJ. ${ }^{6}$

Com a melhora do prognóstico entre as pacientes com LESJ, os reumatologistas pediátricos estão tendo que lidar com questões próprias da adolescência vivida por seus pacientes, entre elas o exercício da sexualidade. A função sexual em jovens com LESJ tem sido ainda pouco estudada, habitualmente com pequena população de pacientes de ambos os gêneros. ${ }^{3,6,8}$ Entretanto, nenhum dos estudos avaliou a função sexual e a saúde reprodutiva e incluiu um grupo controle de adolescentes saudáveis pareado por idade.

O objetivo deste estudo foi avaliar a função sexual e a saúde reprodutiva de adolescentes com LESJ e comparar com grupo controle pareado por idade.

\section{PACIENTES E MÉTODOS}

\section{População do estudo}

Cinquenta e cinco pacientes do sexo feminino com LESJ atendidas consecutivamente, com idades entre 10 e 19 anos, e acompanhados na Unidade de Reumatologia Pediátrica e na Disciplina de Reumatologia do Hospital das Clínicas (HC) da Faculdade de Medicina da Universidade de São Paulo (FMUSP) foram selecionadas para este estudo. Todas as pacientes preencheram os critérios de classificação do Colégio Americano de Reumatologia para o diagnóstico de LESJ. ${ }^{9} \mathrm{O}$ critério de inclusão foi presença de menarca. Nenhuma das pacientes apresentou gravidez atual, diabetes melito associado ao LESJ, uso de contraceptivos hormonais e de gonadotropina (gonadotropin-releasing hormone analogue - $\mathrm{GnRH}-\mathrm{a}$ ) nas infusões com ciclofosfamida intravenosa. Três pacientes foram excluídas por se recusarem a participar do estudo. O grupo final consistiu de 52 pacientes com LESJ. O grupo controle incluiu 52 adolescentes saudáveis pareadas por idade, avaliadas na primeira consulta no Ambulatório Preventivo de Ginecologia do HC-FMUSP. O estudo foi aprovado pelo Comitê de Ética em Pesquisa do HC-FMUSP (CAPPesq 019/04) e o termo de consentimento livre e esclarecido foi assinado por todas as participantes e, quando necessário, por seus respectivos responsáveis.

\section{Métodos}

Os seguintes dados foram coletados de acordo como expresso abaixo:

Dados demográficos e socioeconômico-culturais: dados demográficos (idade de início da doença, tempo de duração do LES e idade atual) e dados socioeconômico-culturais (escolaridade, classe econômica e atividade profissional). As classes socioeconômicas foram avaliadas de acordo a classificação da Associação Brasileira dos Institutos de Pesquisa de Mercados. ${ }^{10}$

Marcos pubertários: Os marcos pubertários foram determinadas de acordo com a idade da primeira menstruação (menarca) e os critérios de alterações propostos por Tanner (características das mamas e distribuição dos pelos pubianos). ${ }^{11}$

Função sexual: A função sexual foi avaliada a partir da história clínica: presença de atividade sexual; idade de início da atividade sexual; realização e número de atividades sexuais no último mês; número de parceiros no último mês; prática de masturbação; presença de desejo e excitação sexual, lubrificação vaginal e orgasmo; presença de insatisfação com a vida sexual; uso de contraceptivo nas relações sexuais (preservativo ou camisinha masculina) e história de gravidez. No presente estudo, a disfunção sexual foi definida como alteração de uma ou mais das seguintes funções sexuais: desejo, excitação, lubrificação vaginal e/ou desempenho reduzidos e/ou ausência de orgasmo (anorgasmia).

Exame ginecológico: Exame clínico sistemático da genitália foi realizado por uma mesma ginecologista e incluiu avaliação da vulva, hímen, vagina e colo uterino. Assim como foi coletado o esfregaço para o teste de Papanicolaou na fase folicular do ciclo menstrual. ${ }^{6}$

Ciclo menstrual: Estes foram avaliados em pelo menos 6 meses consecutivos. Os ciclos normais foram definidos como intervalos variando de 25 a 35 dias com 3 a 7 dias de duração do fluxo, como previamente descrito na população do Brasil. ${ }^{3,5}$ Amenorreia foi definida como interrupção da menstruação por mais de quatro meses consecutivos depois da menarca. Falência ovariana prematura (FOP) foi definida pela presença de amenorreia mantida por mais de 12 meses e valores de FSH $>40$ $\mathrm{UI} / \mathrm{L}$ (técnica de fluoroimmunoensaio usando kits DELPHIA ${ }^{\mathrm{R}}$, WALLAC, Turku, Finlândia). ${ }^{1,3,5,7}$

Citologia cérvico-vaginal: Os esfregaços para o Papanicolaou foram coletados das adolescentes virgens com escova Cytobrush $^{\circledR}$. Esta escova foi inserida na borda do orifício vaginal e suavemente rodada entre $90^{\circ}$ e $180^{\circ}$, e imediatamente rolada sobre o terço externo da lâmina. Nas adolescentes sexualmente ativas, após introdução do espéculo, a citologia foi coletada com escova Cytobrush ${ }^{\circledR}$ e espátula de Ayre. O colo uterino foi 
visualizado e a espátula de Ayre foi inserida no orifício cervical e rodada $360^{\circ}$ com uma leve pressão, depois a escova Cytobrush ${ }^{\circledR}$ foi inserida em dois terços do canal endocervical e rodada entre $90^{\circ}$ e $180^{\circ}$. O material da escova Cytobrush ${ }^{\circledR}$ foi rolado sobre o terço externo da lâmina e o material da espátula de Ayre foi espalhado em fina camada sobre o terço médio da lâmina. Após a fixação por imersão em álcool a 95\%, os esfregaços foram imediatamente transportados ao laboratório. ${ }^{6,12}$

Todos os esfregaços foram avaliados por uma mesma citopatologista do Serviço de Colposcopia do Departamento de Ginecologia da FMUSP que desconhecia o exame ginecológico. Os esfregaços foram avaliados de acordo com o Sistema de Bethesda 2001 em cinco padrões, com presença ou ausência de infecção pelo HPV: normal (alterações celulares benignas), alterações inflamatórias, células escamosas atípicas de significado indeterminado (CEASI), lesões intraepiteliais escamosas de baixo ou alto grau (LIE-BG ou LIE-AG) e carcinoma in situ. As infecções por Trichomonas vaginalis, Candida spp, vaginose bacteriana, Actinomyces spp e vírus herpes simples foram também avaliadas. ${ }^{13}$

Avaliações da atividade do LESJ, dano cumulativo da doença e tratamento: A atividade da doença e o dano cumulativo do LESJ foram avaliados em todos os pacientes no início do estudo, usando os escores Systemic Lupus Erythematosus Disease Activity Index (SLEDAI) ${ }^{14}$ e Systemic Lupus International Collaborating Clinics/ACR-Damage Index (SLICC/ ACR-DI), respectivamente. ${ }^{15}$ Dados referentes ao tratamento com prednisona, cloroquina, ciclofosfamida, azatioprina e metotrexato foram determinados.

\section{Análise estatística}

Resultados foram apresentados em mediana (variação) ou média \pm desvio-padrão para variáveis contínuas e número (\%) para variáveis categóricas. Os resultados foram comparados pelos testes $t$ não pareado e Mann-Whitney para variáveis contínuas para determinar diferenças entre pacientes com LESJ versus controles e entre pacientes com LESJ de acordo com dois grupos: com e sem disfunção sexual. Para as variáveis categóricas, as diferenças foram calculadas pelo teste exato de Fischer. Valores de $\mathrm{P}<0,05$ foram considerados estatisticamente significativos.

\section{RESULTADOS}

\section{Adolescentes com LESJ versus controles saudáveis}

As médias de idade atual e de anos de escolaridade foram similares entre adolescentes com LESJ e grupo controle (16,7 $\pm 1,94$ versus $16,13 \pm 2,16$ anos, $\mathrm{P}=0,92 ; 9,51 \pm 1,78$ versus $9,7 \pm 1,62$ anos, $\mathrm{P}=0,57$; respectivamente). Assim como os percentuais de classe socioeconômica $\mathrm{C}$ ou $\mathrm{D}$ e a realização de atividade profissional foram semelhantes entre os grupos. Os dados demográficos e socioeconômico-culturais, marcos pubertários, função sexual, ciclo menstrual e citologia cérvico-vaginal de adolescentes com LESJ versus controles estão na Tabela 1 .

A média de idade da menarca foi estatisticamente maior nas pacientes com LESJ versus controles (12,82 $\pm 1,62$ versus $11,54 \pm 1,45$ anos, $\mathrm{P}=0,00004)$. Todas as pacientes e controles apresentaram predomínio dos estágios pubertários de Tanner M4P4 e M5P5 ${ }^{11}$ (75\% versus $\left.73 \%, \mathrm{P}=1,0\right)$.

Com relação à função sexual, a frequência de início de atividade sexual foi estatisticamente menor nas pacientes com LESJ em relação ao grupo controle (23\% versus $60 \%$, $\mathrm{P}=0,0003$ ). Disfunção sexual (presença de redução do desejo, excitação, lubrificação vaginal e/ou desempenho e/ou anorgasmia) e insatisfação com a vida sexual foram relatadas em 58\% das pacientes com LESJ versus 23\% dos controles $(\mathrm{P}=0,03)$. Além disto, os percentuais de reduções de lubrificação vaginal e desempenho no ato sexual, assim como anorgasmia foram estatisticamente maiores nas pacientes com LESJ comparadas ao grupo controle (50\% versus $16 \%$, $\mathrm{P}=0,046 ; 58 \%$ versus $23 \%, \mathrm{P}=0,03 ; 50 \%$ versus $16 \%, \mathrm{P}=$ 0,046 ; respectivamente). Houve tendências estatísticas entre maiores frequências de diminuições do desejo e excitação nas adolescentes com LESJ e controles (33\% versus $10 \%, \mathrm{P}=$ 0,$08 ; 33 \%$ versus $10 \%, \mathrm{P}=0,08$; respectivamente). Não houve diferença estatística entre idade da primeira atividade sexual, número de atividades sexuais e de parceiros no último mês, bem como uso de preservativo masculino e gravidez prévia nos dois grupos estudados $(\mathrm{P}>0,05)$ (Tabela 1$)$.

Irregularidade de duração e/ou intervalo do ciclo menstrual e amenorreia foram similares entre pacientes com LESJ e controles $(23 \%$ versus $11 \%, \mathrm{P}=0,19 ; 6 \%$ versus $0 \%, \mathrm{P}=$ 0,19 ; respectivamente). Nenhuma das pacientes apresentou FOP. Citologia cérvico-vaginal inflamatória ocorreu em 48\% das pacientes com LESJ versus $46 \%$ controles $(\mathrm{P}=1,0)$. Apenas $2 \%$ das pacientes com LESJ e $4 \%$ dos controles tiveram displasia cervical (LIE-BP) com infecção por $\operatorname{HPV}(\mathrm{P}=1,0)$. Um aspecto interessante foi uma maior frequência, estatisticamente significativa, de candidíase vaginal nas pacientes com LESJ versus controles ( $14 \%$ versus $0 \%, \mathrm{P}=0,01)$. Não houve diferença estatística entre a prevalência de vaginose por Gardnerella vaginalis nos dois grupos estudados $(\mathrm{P}=$ 0,11) (Tabela 1). 
Tabela 1. Dados demográficos e socioeconômicoculturais, marcos pubertários, função sexual, ciclo menstrual e citologia cérvico-vaginal de adolescentes com lúpus eritematoso sistêmico (LESJ) versus controles

\begin{tabular}{|c|c|c|c|}
\hline Variáveis & $\begin{array}{c}\text { LESJ } \\
(\mathrm{n}=52)\end{array}$ & $\begin{array}{l}\text { Controles } \\
(n=52)\end{array}$ & $\mathrm{P}$ \\
\hline \multicolumn{4}{|l|}{$\begin{array}{l}\text { Dados demográficos e } \\
\text { socioeconômico-culturais }\end{array}$} \\
\hline Idade atual, anos $* * *$ & $16,7 \pm 1,94$ & $16,13 \pm 2,16$ & 0,92 \\
\hline Escolaridade, anos $* * *$ & $9,51 \pm 1,78$ & $9,7 \pm 1,62$ & 0,57 \\
\hline Classe econômica C ou D * & $38(73)$ & $41(79)$ & 0,64 \\
\hline Atividade profissional * & $3(6)$ & $10(19)$ & 0,07 \\
\hline \multicolumn{4}{|l|}{ Marcos pubertários } \\
\hline Idade da menarca, anos $* * *$ & $12,82 \pm 1,62$ & $11,54 \pm 1,45$ & 0,00004 \\
\hline $\begin{array}{l}\text { Estágio pubertário de } \\
\text { Tanner M4P4 ou M5P5 * }\end{array}$ & $39(75)$ & $38(73)$ & 1,0 \\
\hline \multicolumn{4}{|l|}{ Função sexual } \\
\hline Atividade sexual * & $12 / 52(23)$ & $31 / 52(60)$ & 0,0003 \\
\hline $\begin{array}{l}\text { Idade de início da atividade } \\
\text { sexual, anos } * * *\end{array}$ & $15,3 \pm 1,72$ & $14,87 \pm 1,99$ & 0,48 \\
\hline $\begin{array}{l}\text { Atividade sexual no } \\
\text { último mês } *\end{array}$ & 10/12 (83) & 28/31 (90) & 0,60 \\
\hline $\begin{array}{l}\text { Número de atividades } \\
\text { sexuais no último mês } * * *\end{array}$ & $4,33 \pm 4,73$ & $5,45 \pm 4,02$ & 0,44 \\
\hline $\begin{array}{l}\text { Número parceiros } \\
\text { no último mês ** }\end{array}$ & $1(1-5)$ & $1(1-10)$ & 1,0 \\
\hline Masturbação * & $0 / 12(0)$ & $2 / 31(6)$ & 1,0 \\
\hline Disfunção sexual * & 7/12 (58) & $7 / 31(23)$ & 0,03 \\
\hline Desejo reduzido * & $4 / 12(33)$ & $3 / 31(10)$ & 0,08 \\
\hline Excitação reduzida * & $4 / 12(33)$ & $3 / 31(10)$ & 0,08 \\
\hline Lubrificação reduzida * & $6 / 12(50)$ & $5 / 31(16)$ & 0,046 \\
\hline Desempenho reduzido * & $7 / 12(58)$ & $7 / 31(23)$ & 0,03 \\
\hline Anorgasmia * & $6 / 12(50)$ & $5 / 31(16)$ & 0,046 \\
\hline $\begin{array}{l}\text { Insatisfação com a } \\
\text { vida sexual * }\end{array}$ & $7 / 12(58)$ & $7 / 31(23)$ & 0,035 \\
\hline $\begin{array}{l}\text { Uso de preservativo } \\
\text { masculino * }\end{array}$ & $10 / 12(83)$ & 28/31 (90) & 0,60 \\
\hline Gravidez prévia * & $1 / 12(8)$ & 4/31 (13) & 1,0 \\
\hline \multicolumn{4}{|l|}{ Ciclo menstrual } \\
\hline $\begin{array}{l}\text { Irregularidades de duração } \\
\text { e/ou intervalo * }\end{array}$ & $12(23)$ & $6(11)$ & 0,19 \\
\hline Amenorreia * & $3(6)$ & $0(0)$ & 0,24 \\
\hline \multicolumn{4}{|l|}{ Citologia cérvico-vaginal } \\
\hline Normal * & $26(50)$ & $26(50)$ & 1,15 \\
\hline Inflamatória * & $25(48)$ & $24(46)$ & 1,0 \\
\hline LIE-BG * & $1(2)$ & $2(4)$ & 1,0 \\
\hline Candidíase vaginal * & $7(14)$ & $0(0)$ & 0,01 \\
\hline $\begin{array}{l}\text { Vaginose por Gardnerella } \\
\text { vaginallis * }\end{array}$ & $0(0)$ & $4(8)$ & 0,11 \\
\hline Infecção por HPV * & $1(2)$ & $2(4)$ & 1,0 \\
\hline $\begin{array}{l}\text { Condiloma acuminado } \\
\text { por HPV * }\end{array}$ & $0(0)$ & $2(4)$ & 0,49 \\
\hline
\end{tabular}

\section{Adolescentes com LESJ com e sem alguma disfunção sexual relatada na história clínica}

Os dados demográficos e socioeconômico-culturais, marcos pubertários, função sexual, ciclo menstrual e citologia cérvicovaginal das 12 adolescentes com LESJ com atividade sexual de acordo com a presença ou não de disfunção sexual estão na Tabela 2 .

As medianas da idade de início da doença (12 versus 15 anos, $\mathrm{P}=0,18$ ), idade atual (18 versus 17 anos, $\mathrm{P}=0,795)$ e anos de escolaridade (11 versus 11 anos, $\mathrm{P}=0,66$ ) foram similares entre as pacientes com LESJ com e sem disfunção sexual. Não houve diferença estatística entre tempo de duração da doença, idade da menarca, escolaridade, classes socioeconômicas, atividade profissional e estágios pubertários de Tanner nos dois grupos estudados $(\mathrm{P}>0,05)$ (Tabela 2).

Não houve diferença estatística em relação às frequências de: uso de preservativo masculino, gravidez prévia e irregularidades do ciclo menstrual entre pacientes com LESJ que relataram disfunção sexual versus as que tinham função normal. Além disso, nos dois grupos estudados não ocorreram diferenças estatisticamente significativas entre as frequências da citologia cérvico-vaginal: normal, inflamatória, displasia (LIE-BG), candidíase vaginal, vaginose por Gardnerella vaginalis e condiloma acuminado por HPV $(\mathrm{P}>0,05)$ (Tabela 2$)$.

A Tabela 3 inclui atividade, dano cumulativo e tratamento de adolescentes com LESJ com disfunção versus sem disfunção sexual. As medianas do SLEDAI [4 (0-19) versus 6 (2-16), $\mathrm{P}=0,41$ ] e SLICC/ACR-DI [0 (0-1) versus 0 (0-1), $\mathrm{P}=0,8]$ foram similares nas pacientes com LESJ com disfunção sexual comparadas aos que tinham função normal, assim como as frequências de SLEDAI $>4$ e SLICC/ACR-DI $>1$ $(\mathrm{P}>0,05)$. As frequências do uso de prednisona, cloroquina e imunossupressores (ciclofosfamida, azatioprina e metotrexato), assim como as doses cumulativas de prednisona e cloroquina também foram similares nos dois grupos estudados $(\mathrm{P}>0,05)$ (Tabela 3).

\section{DISCUSSÃO}

Este é o primeiro estudo que avaliou universalmente a função sexual e a saúde reprodutiva de jovens do sexo feminino com LESJ e identificou disfunção sexual global e específica em adolescentes com lúpus quando comparadas a um grupo controle com mesmo estágio pubertário, e confirmou atraso da menarca em adolescentes com lúpus registrados em estudos anteriores.

Neste estudo a função sexual feminina incluiu características da história clínica, que compreende aspectos de desejo, 
Tabela 2. Dados demográficos e socioeconômicoculturais, marcos pubertários, função sexual, ciclo menstrual e citologia cérvico-vaginal de adolescentes com lúpus eritematoso sistêmico (LESJ) com versus sem disfunção sexual

\begin{tabular}{|c|c|c|c|}
\hline Variáveis & $\begin{array}{c}\text { LESJ com } \\
\text { disfunção } \\
\text { sexual }(\mathbf{n}=7)\end{array}$ & $\begin{array}{c}\text { LESJ sem } \\
\text { disfunção } \\
\text { sexual }(n=5)\end{array}$ & $\mathbf{P}$ \\
\hline \multicolumn{4}{|c|}{ Dados demográficos e socioeconômico-culturais } \\
\hline $\begin{array}{l}\text { Idade de início do } \\
\text { LESJ, anos } * *\end{array}$ & $12(10-16)$ & $15(14-17)$ & 0,18 \\
\hline $\begin{array}{l}\text { Tempo de duração } \\
\text { do LESJ, anos ** }\end{array}$ & $3(1-6)$ & $4(2-5)$ & 0,084 \\
\hline Idade atual, anos $* *$ & $18(15-19)$ & $17(15-18)$ & 0,41 \\
\hline Escolaridade, anos ** & $11(7-11)$ & $11(9-11)$ & 0,66 \\
\hline Classe econômica C ou D * & $6(86)$ & $4(80)$ & 1,0 \\
\hline Atividade profissional * & $1(14)$ & $0(0)$ & 1,0 \\
\hline \multicolumn{4}{|l|}{ Marcos pubertários } \\
\hline Idade da menarca, anos $* * *$ & $13,57 \pm 1,90$ & $11,6 \pm 1,14$ & 0,063 \\
\hline $\begin{array}{l}\text { Estágio pubertário de } \\
\text { Tanner M4P4 ou M5P5 * }\end{array}$ & $7(100)$ & $4(80)$ & 1,0 \\
\hline \multicolumn{4}{|l|}{ Função sexual } \\
\hline $\begin{array}{l}\text { Idade de início da } \\
\text { atividade sexual, anos ** }\end{array}$ & $16(14-17)$ & $14(13-18)$ & 0,32 \\
\hline $\begin{array}{l}\text { Atividade sexual no } \\
\text { último mês * }\end{array}$ & $6(86)$ & $4(80)$ & 1,0 \\
\hline $\begin{array}{l}\text { Número de atividades } \\
\text { sexuais no último mês ** }\end{array}$ & $4(0-16)$ & $2(0-6)$ & 0,32 \\
\hline $\begin{array}{l}\text { Número parceiros } \\
\text { no último mês ** }\end{array}$ & $1(1-5)$ & 1 & 0,39 \\
\hline $\begin{array}{l}\text { Uso de preservativo } \\
\text { masculino } *\end{array}$ & $5(71)$ & $5(100)$ & 0,46 \\
\hline Gravidez prévia * & $1(14)$ & $0(0)$ & 1,0 \\
\hline \multicolumn{4}{|l|}{ Ciclo menstrual } \\
\hline $\begin{array}{l}\text { Irregularidades da duração } \\
\text { e/ou intervalo * }\end{array}$ & $2(29)$ & $3(60)$ & 0,55 \\
\hline Amenorreia * & $0(0)$ & $0(0)$ & 1,0 \\
\hline \multicolumn{4}{|l|}{ Citologia cérvico-vaginal } \\
\hline Normal * & $3(43)$ & $0(0)$ & 0,20 \\
\hline Inflamatória * & $3(43)$ & $5(100)$ & 0,08 \\
\hline LIE-BG * & $1(14)$ & $0(0)$ & 1,0 \\
\hline Candidíase vaginal * & $1(14)$ & $1(20)$ & 1,0 \\
\hline $\begin{array}{l}\text { Vaginose por Gardnerella } \\
\text { vaginallis * }\end{array}$ & $0(0)$ & $0(0)$ & 1,0 \\
\hline Infecção por HPV * & $1(14)$ & $0(0)$ & 1,0 \\
\hline $\begin{array}{l}\text { Condiloma acuminado } \\
\text { por HPV } *\end{array}$ & $0(0)$ & $0(0)$ & 1,0 \\
\hline
\end{tabular}

*Valores são expressos em n (\%); ** mediana (valor máximo - valor mínimo);

***média \pm desvio-padrão; $M=$ mamas; $P=$ pelos pubianos;

$\mathrm{LIE}-\mathrm{BG}=$ lesão intraepitelial de baixo grau; HPV = papilomavírus humano.
Tabela 3. Atividade, dano cumulativo e tratamento de adolescentes do sexo feminino com lúpus eritematoso sistêmico (LESJ) com versus sem disfunção sexual

\begin{tabular}{|c|c|c|c|}
\hline Variáveis & $\begin{array}{c}\text { LESJ com } \\
\text { disfunção } \\
\text { sexual }(\mathbf{n}=7)\end{array}$ & $\begin{array}{c}\text { LESJ sem } \\
\text { disfunção } \\
\text { sexual }(n=5)\end{array}$ & $\mathbf{P}$ \\
\hline \multicolumn{4}{|c|}{ Atividade e dano cumulativo do LESJ } \\
\hline SLEDAI $^{* *}$ & $4(0-19)$ & $6(2-16)$ & 0,41 \\
\hline SLEDAI $>4^{*}$ & $4(57)$ & $3(60)$ & 1,0 \\
\hline SLICC/ACR-DI ${ }^{* *}$ & $0(0-1)$ & $0(0-1)$ & 0,8 \\
\hline SLICC/ACR-DI $>1^{*}$ & $1(14)$ & $1(20)$ & 1,0 \\
\hline \multicolumn{4}{|l|}{ Terapêutica do LESJ } \\
\hline Prednisona * & $7(100)$ & $5(100)$ & 1,0 \\
\hline $\begin{array}{l}\text { Dose cumulativa, } \\
\text { gramas }^{* *}\end{array}$ & $23(10,55-36)$ & $22,4(2,34-24)$ & 0,29 \\
\hline Cloroquina ${ }^{*}$ & $5(71)$ & $5(100)$ & 0,46 \\
\hline $\begin{array}{l}\text { Dose cumulativa, } \\
\text { gramas }^{* *}\end{array}$ & $264(45-428)$ & $64,4(9-274)$ & 0,25 \\
\hline Ciclofosfamida * & $2(29)$ & $0(0)$ & 0,46 \\
\hline Azatioprina * & $2(29)$ & $0(0)$ & 0,46 \\
\hline Metotrexato * & $1(14)$ & $0(0)$ & 1,0 \\
\hline
\end{tabular}

* Valores são expressos em n (\%); *^ ou mediana (valor máximo - valor mínimo);

SLEDAl - Systemic Lupus Erythematosus Disease Activity Index,

SLICC/ACR-DI - Systemic Lupus International Collaborating Clinics/ACR Damage Index.

excitação, lubrificação vaginal, desempenho, orgasmo, além da satisfação com a vida sexual como um todo. ${ }^{3,16}$ Os raros estudos que avaliaram a função sexual de pacientes com lúpus, habitualmente, não tinham grupo controle e geralmente incluíram pacientes adultos de ambos os sexos. ${ }^{3,6,8,17-20}$ Além do mais, nenhum destes realizou uma avaliação concomitante do ciclo menstrual e da citologia cérvico-vaginal.

Stein et al. ${ }^{17}$ evidenciaram que $4 \%$ das mulheres e dos homens adultos com LES apresentaram disfunções sexuais por dados de história clínica. Folomeev \& Alekberova ${ }^{18}$ identificaram uma alta frequência de disfunção sexual/erétil em $35 \%$ homens com LES. A partir de uma entrevista estruturada em mulheres adultas, Curry et al. ${ }^{19}$ observaram frequências reduzidas de atividade sexual, lubrificação vaginal e satisfação sexual em lúpus versus grupo controle pareado, como observado nas adolescentes com LESJ do presente estudo. Britto et al. ${ }^{8}$ avaliaram aspectos da sexualidade em 178 adolescentes com doenças reumatológicas crônicas, entretanto apenas 15 desses pacientes tinham LESJ. Atividade sexual ocorreu em 21\% dos adolescentes do sexo masculino e $60 \%$ do sexo feminino.

A disfunção sexual na adolescente do sexo feminino com lúpus é multifatorial e pode ocorrer pela própria atividade 
da doença ou pelos medicamentos, como corticosteroides e imunossupressores. ${ }^{3}$ No presente estudo não foi demonstrado associação entre disfunção sexual e atividade do lúpus, dano cumulativo causado pela doença ou uso de medicações. Isso pode ser explicado pelo pequeno número de pacientes em cada grupo o que pode comprometer a análise estatística, ou seja, podemos estar diante de um erro Beta, quando existe diferença real entre os grupos, mas o tamanho da amostra não foi suficiente para demonstrar a diferença. No entanto, a função sexual é um parâmetro complexo para ser analisado e pacientes jovens com lúpus podem querer omitir dos pais e médicos que já iniciaram atividade sexual (por medo, vergonha etc.). Além disto, vários outros fatores não abordados no estudo também poderiam influenciar a função sexual, tais como: fatores próprios da adolescência, redução da autoestima, estresse da doença crônica, depressão associada e compreensão do parceiro no ato sexual.

Um aspecto relevante observado na pesquisa é o de que as pacientes com LESJ iniciaram atividade sexual com a mesma idade das adolescentes saudáveis (mediana de 16 anos). Esta idade também foi semelhante à encontrada em um estudo com adolescentes com epilepsia (mediana de 15 anos) ${ }^{21}$ realizado pelo mesmo grupo do presente estudo e reforça que, a contracepção para prevenção das doenças sexualmente transmissíveis, incluindo síndrome da imunodeficiência adquirida (Aids) e HPV ${ }^{3,6}$ deve ser uma preocupação cada vez mais precoce nas adolescentes que iniciam atividade sexual. Além disto, a atividade sexual desprotegida tem aumentado os números de gestações indesejadas e não programadas entre essas pacientes, conforme constatado em recente estudo multicêntrico nacional realizado em 12 Serviços de Reumatologia Pediátrica. ${ }^{22}$ Gravidez indesejada foi também observada em $8 \%$ das pacientes com lúpus sexualmente ativas do presente estudo.

As utilizações de preservativo masculino em todas as atividades sexuais e da contracepção de emergência são prioritárias em adolescentes com lúpus que receberão ciclofosfamida e metotrexato. Kaufman, 2008, sugere que a contracepção de emergência (com levonorgestrel 1,5 mg) deva ser utilizada imediatamente nas pacientes com LESJ, principalmente nas primeiras 72 horas, após uma atividade sexual sem utilização de preservativo ou sua ruptura no ato sexual. ${ }^{23}$ Nenhuma das pacientes deste estudo utilizou este método anticoncepcional ou outro contraceptivo hormonal.

Outro aspecto relevante foi avaliação de parâmetros da saúde reprodutiva neste estudo. Apesar de uma maior frequência de irregularidades menstruais e amenorreia nas pacientes com LESJ, não houve diferença estatística entre os grupos, diferindo de um recente estudo do nosso grupo. ${ }^{5}$ Nenhuma das nossas pacientes apresentou falência ovariana prematura, mesmo utilizando terapia com imunossupressores. Adolescentes do sexo masculino com LESJ que utilizaram ciclofosfamida também podem ter azoospermia e disfunção definitiva das células testiculares de Sertoli. ${ }^{20,24,25}$

Candidíase vaginal foi mais frequente nas adolescentes com lúpus, conforme evidenciado em outro estudo. ${ }^{6}$ Isto reforça a importância da pesquisa rotineira desta infecção, pois a infecção fúngica foi uma das principais causas de óbito em pacientes internados com LESJ no nosso serviço terciário de Reumatologia Pediátrica. ${ }^{26}$

Este estudo incluiu uma ampla avaliação da função sexual e da saúde reprodutiva que pode ser utilizada na prática do reumatologista pediátrico. Entretanto, serão necessários futuros estudos utilizando amostras maiores, instrumentos mais específicos de função sexual ${ }^{16} \mathrm{e}$ validados na população brasileira de adolescentes com LESJ de ambos os sexos, além da avaliação global da função sexual nos seus parceiros.

O presente estudo identificou disfunção sexual em adolescentes do sexo feminino com LESJ. Aspectos relacionados à sexualidade necessitam uma atenção especial dos profissionais de saúde que atendem adolescentes com lúpus, propiciando melhor qualidade de vida das pacientes e de seus parceiros.

\section{AGRADECIMENTOS}

Os autores agradecem à Prof ${ }^{a}$. Thelma Suely Okay pela realização das dosagens hormonais e ao Dr. Ulysses DóriaFilho pelo auxílio à análise estatística. 


\section{REFERÊNCIAS}

\section{REFERENCES}

1. Silva CA, Brunner HI. Gonadal functioning and preservation of reproductive fitness with juvenile systemic lupus erythematosus. Lupus 2007; 16:593-9.

2. Silva CA, Moraes AJ. Avaliação da função gonadal em adolescentes e jovens com doenças reumáticas. Rev Paulista Reumatol 2008; 7:6-9.

3. Silva CA, Leal MM, Campos LM et al. Aspectos da sexualidade e gravidez de adolescentes e adultos jovens com lúpus eritematoso sistêmico (LES). Rev Bras Reumatol 2001; 41:213-9.

4. Silva CA, Leal MM, Leone $\mathrm{C}$ et al. Gonadal function in adolescents and young women with juvenile systemic lupus erythematosus. Lupus 2002; 11:419-25.

5. Medeiros P, Febrônio M, Bonfá E, Borba E, Takiuti A, Silva C. Menstrual and hormonal alterations in juvenile systemic lupus erythematosus. Lupus 2009; 18:38-43.

6. Febronio MV, Pereira RM, Bonfa E et al. Inflammatory cervicovaginal cytology is associated with disease activity in juvenile systemic lupus erythematosus. Lupus 2007; 16:430-5.

7. Silva CA, Hilário MO, Febrônio MV et al. Risk factors for amenorrhea in juvenile systemic lupus erythematosus (JSLE): a Brazilian multicentre cohort study. Lupus 2007; 16:531-6.

8. Britto MT, Rosenthal SL, Taylor J, Passo MH. Improving rheumatologists screening for alcohol use and sexual activity. Arch Pediatr Adolesc Med 2000; 154:478-83.

9. Hochberg MC. Updating the American College of Rheumatology revised criteria for the classification of systemic lupus erythematosus. Arthritis Rheum 1997; 40:1725.

10. Almeida PM, Wickerrhauser H. Critério de classe econômica da Associação Brasileira de Anunciantes (ABA) e Associação Brasileira dos Institutos de Pesquisa de Mercado (ABIPEME) 1991, pp. 1-29.

11. Tanner JM. Growth at adolescence, 2 ed., Oxford, Blackwell Scientific Publications, 1962.

12. Fokke HE, Salvatore CM, Schipper MEI, Bleker OP. A randomized trial of three methods of obtaining Papanicolaou smears. Eur J Obstet Gynecol Reprod Bio 1993; 48:103-6.

13. Solomon D, Davey D, Kurman R et al. The 2001 Bethesda System: terminology for reporting results of cervical cytology. JAMA 2002; 287:2114-9.

14. Brunner HI, Silverman ED, To T, Bombardier C, Feldman BM. Sensitivity of the Systemic Lupus Erhytematosus Disease Activity Index, British Isles Lupus Assessment Group Index, and Systemic Lupus Activity Measure in the evaluation of clinical change in childhood-onset systemic lupus erythematosus. Arthrits Rheum 1999; 42:1354-60.

15. Brunner HI, Silverman ED, To T, Bombardier C, Feldman BM. Risk factors for damage in childhood-onset systemic lupus erythematosus: cumulative disease activity and medication use predict disease damage. Arthritis Rheum 2002; 46:436-44. 
16. Quaresma MR, Goldsmith CH, Lamont J, Ferraz MB. Assessment of sexual function in patients with rheumatic disorders: a critical appraisal. J Rheumatol 1997; 24:1673-6.

17. Stein H, Walters K, Dillon A, Schulzer M. Systemic lupus erythematosus - a medical and social profile. J Rheumatol 1986; 13:570-5.

18. Folomeev M, Alekberova Z. Impotence in systemic lupus erythematosus. J Rheumatol 1990; 17:117-9.

19. Curry SL, Levine SB, Corty E, Jones PK, Kurit DM. The impact of systemic lupus erythematosus on women's sexual functioning. J Rheumatol 1994; 21:2254-60.

20. Silva CA, Hallak J, Pasqualotto FF, Barba MF, Salto Ml, Kiss MH. Gonadal function in adolescents and young men with systemic lupus erythematosus. J Rheumatol 2002; 29:2000-5.

21. de Vincentiis S, Febrônio MV, da Silva CA et al. Sexuality in teenagers with epilepsy. Epilepsy Behav 2008; 13:703-6.
22. Silva CA, Hilario MO, Febronio MV et al. Pregnancy outcome in juvenile systemic lupus erythematosus: a Brazilian multicenter cohort study. J Rheumatol 2008; 35:1414-8.

23. Kaufman M. Pregnant adolescents and youth with systemic lupus erythematosus: can new data inform our approach to young women with SLE? J Rheumatol 2008; 35:1240-1.

24. Soares PM, Borba EF, Bonfa E, Hallak J, Corrêa AL, Silva CA. Gonad evaluation in male systemic lupus erythematosus. Arthritis Rheum 2007; 56:2352-61.

25. Suehiro RM, Borba EF, Bonfa E et al. Testicular Sertoli cell function in male systemic lupus erythematosus. Rheumatology (Oxford) 2008; 47:1692-7.

26. Faco MM, Leone C, Campos LM, Febrônio MV, Marques HH, Silva CA. Risk factors associated with the death of patients hospitalized for juvenile systemic lupus erythematosus. Braz J Med Biol Res 2007; 40:993-1002. 